\title{
Prevalence and associated factors of knee osteoarthritis in a rural Chinese adult population: an epidemiological survey
}

Yuan Liư ${ }^{2 \dagger}$, Haifeng Zhang ${ }^{3+}$, Ningxia Liang ${ }^{3}$, Weimin Fan ${ }^{1}$, Jun Li ${ }^{2}$, Zuhu Huang ${ }^{2}$, Zhijian Yin ${ }^{4}$, Zhijun Wu ${ }^{4}$ and Jun $\mathrm{Hu}^{1 *}$

\begin{abstract}
Background: The exact pathogenic mechanism of knee osteoarthritis $(O A)$ is still unknown. With the exception of clinical treatment to alleviate symptoms, or total knee replacement, there is currently no effective treatment method. Consequently, an in-depth etiological and epidemiological study of knee OA can provide clues for diagnosis, treatment and scientific research, and will ultimately have a beneficial effect on public health.

Methods: A cross-sectional community study in the rural village of Gaoyou was conducted in 3428 Chinese adults (aged $\geq 40$ years). Subjects completed an interviewer-administered questionnaire, evaluating knee pain and associated disability, analgesia, use of health services, past medical history, walking, income, smoking, and use of oral contraceptives, and standardized weight-bearing knee radiographs were obtained. Patient demographic characteristics and biochemical parameters were recorded.
\end{abstract}

Results: Single-factor regression analysis indicated that age, overweight, central adiposity, high low-density lipoprotein cholesterol (LDLC), high total cholesterol (TC), high triglycerides (TG), dyslipidemia, hypertension and low income were the associated factors for knee OA in females; age, high LDLC, hypertension, low income and frequent walking were the associated factors for knee OA in males. Interestingly, male heavy smokers were less likely to develop severe knee OA compared with non-smokers. Stepwise logistic regression analysis indicated that age and overweight were the associated factors for knee OA for all individuals. Although central adiposity, high LDLC, high TC, high TG, dyslipidemia, hypertension and low income appeared to be related to knee OA in females according to univariate analysis, these factors were not identified in stepwise logistic regression analysis. In addition although age, high LDLC, hypertension and frequent walking were also the associated factors for knee OA in males by stepwise logistic regression analysis, smoking as a protective factor was not identified in this analysis.

Conclusions: In this study, aging, obesity, frequent walking, low income and relevant multiple metabolic disorders were the associated factors for knee OA. Smoking might be associated with a lower prevalence of OA in male smokers according to univariate analysis. A retrospective association of smoking with OA may constitute an important etiologic clue, but further well-designed, large-scale prospective controlled trials are required to confirm these findings.

Keywords: Knee osteoarthritis, Epidemiology, Prevalence, Metabolic syndrome, Smoking

\footnotetext{
* Correspondence: junhu89@vip.sina.com

${ }^{\dagger}$ Equal contributors

${ }^{1}$ Department of Orthopedics, The First Affiliated Hospital of Nanjing Medical

University, Guang Zhou Road 300, Nanjing 210029, China

Full list of author information is available at the end of the article
} 


\section{Background}

Osteoarthritis (OA) is the most common joint disease worldwide, and primarily affects the knees, hips, hands, and spine. It is a leading cause of disability among older individuals aged above 40 years. Besides affecting patients' activity and quality of life, OA will further cause depression and anxiety, as well as a great economic burden [1].

To date, most large population-based epidemiological studies evaluating the disease prevalence of OA have been performed in Europe or North America although it has been estimated that by 2050, almost four fifths of the world's older population (65 years and older) will be living in less-developed regions of the world [2]. To start to address this disparity, several reports on the prevalence of knee OA in China have been conducted. One study reported that the prevalence of radiographic knee OA (42.8 \%) and symptomatic knee OA (15.0\%) in elderly women over 60 years of age in the urban district of Beijing was higher than that in elderly American women of the same age group. In contrast the prevalence of radiographic knee OA and symptomatic knee $\mathrm{OA}$ in Chinese males was similar to that in American males [2]. In another study the population over 50 years of age in Wuchuan County of Inner Mongolia was analyzed, and their data were compared with those from Beijing and Framingham (Massachusetts, USA). The results indicated that the prevalence of symptomatic knee OA in the residents of rural Wuchuan County was higher than that in the urban residents of Beijing or Framingham. However most respondents in Wuchuan County were heavy laborers and this county is located in Inner Mongolia, with a unique natural environment. As a result, the prevalence might be unrepresentative $[3,4]$.

Obesity, hypertension, dyslipidemia, diabetes and insulin resistance tend to cluster into so-called metabolic syndrome (MS). There is growing evidence suggesting that metabolic syndrome (MS) is a risk factor for the development of OA [5-8]. Among the 1334 white patients in a study by Gandhi et al., 114 (8.5 \%) had MS as compared with 3 of 36 (8.3\%) blacks and 18 of 90 (20\%) Asians. Adjusted analysis showed that those of Asian ethnicity had double the risk of MS compared with those of other ethnicities. MS is a risk factor for OA, and Asians demonstrate a greater prevalence of MS compared with whites and blacks in this population [9]. However, there are few publications which mention the associated factors, such as metabolic diseases, for knee OA in the Chinese population. Thus, in order to facilitate the epidemiological study of knee OA in China, the rural population of Gaoyou City of Jiangsu Province (located in the economic belt along the Yangtze Delta, $300 \mathrm{~km}$ from Shanghai) was sampled in this study.

We performed a cross-sectional study in the Han population above 40 years of age in the rural areas of
Gaoyou City to investigate the prevalence of knee OA in males and females and its distribution in each age group. Relevant associated factors, including age, overweight, central adiposity, high low-density lipoprotein cholesterol (LDLC), high total cholesterol (TC), high triglycerides (TG), low high-density lipoprotein (HDL), dyslipidemia, hypertension, diabetes, hyperuricemia, income level, walking habit, smoking habit, and use of oral contraceptives were analyzed. We aimed to provide basic data on the epidemiological features of knee OA in a Chinese population, thus providing a scientific basis for preventing and treating osteoarthritis.

\section{Patients and methods}

Proportionately stratified random sampling was used to select a representative sample from the towns of Baqiao and Songqiao in Gaoyou, a rural area $300 \mathrm{~km}$ north of Shanghai, China. The sample was stratified by sex and age based on population data obtained from the local authorities. Ultimately, 5000 subjects in Baqiao and 1000 subjects in Songqiao aged 18 to 75 years old were selected and invited to participate in the study. In the period from January to May 2010, 4536 subjects participated in the present study, including 3918 subjects in Baqiao and 618 subjects in Songqiao; the response rates were 78.4 and $61.8 \%$, respectively. Of these 4536 respondents, 3428 subjects aged 40-74 years old were selected and invited to participate in the study of prevalence and associated factors of knee osteoarthritis. The protocol was approved by the Ethics Committee of the First Affiliated Hospital with Nanjing Medical University. Informed consent was obtained and signed by each participant $[10,11]$.

Trained health professionals administered the survey questionnaires because it was anticipated that many study participants would be illiterate. All interviewers, clinical examiners, and radiography technicians were trained under the supervision of the study's chief investigators. Participants were interviewed at their homes or site of identification. Trained health professional interviewers administered a standardized questionnaire that focused on joint symptoms, previous diagnoses of arthritis, possible associated factors for OA, and more detailed information on general health status, height, weight, smoking habits, medication, income, use of hormones, specific physical loads from occupation and housework, and sports activities. At the end of the interview, participants were invited to the central examination site for a clinical examination, laboratory testing, and radiography. Transportation to the hospital was provided. All biochemical parameter measures were ascertained at time of study visit using a standard protocol rather than reliance on existing medical and other records.

A weight-bearing posteroanterior view radiograph was taken of both knees strictly according to a validated 
acquisition protocol $[3,12]$. Radiographs were read by the study's chief investigator using the Osteoarthritis Research Society International atlas, and Kellgren/ Lawrence $(\mathrm{K} / \mathrm{L})$ grades (range 0-4) were also assigned. We used the same definitions to define cases of symptomatic knee OA as were used in previous studies [2, 3, 13, 14]. Symptomatic knee OA was defined as having at least one knee with both a K/L grade 2 and a positive response to the question, "In the past 12 months, have you had knee pain lasting most days for at least a month?" Knee OA In this study means symptomatic knee OA.

Standing height was measured with a fixed stadiometer calibrated in centimeters. Weight was measured to the nearest $0.1 \mathrm{~kg}$ using a balance beam scale with subjects wearing light clothing and no shoes. Body mass index (BMI) was calculated as weight in kilograms divided by height squared in meters. Overweight was defined as BMI $\geq 25 \mathrm{~kg} / \mathrm{m}^{2}$ in both men and women. Waist circumference was measured (in centimeters) between the lowest rib margin and the iliac crest [15]. Central adiposity was defined as waist circumference $\geq 90 \mathrm{~cm}$ in men or $\geq 80 \mathrm{~cm}$ in women [16]. A number of laboratory tests, such as LDLC, TC, TG, HDL, fasting plasma glucose, and uric acid were performed. High LDLC was defined as $\geq 4.14 \mathrm{mmol} / \mathrm{L}$. High TC was defined as $\geq 6.12 \mathrm{mmol} / \mathrm{L}$. High TG was defined as $\geq 2.26 \mathrm{mmol} / \mathrm{L}$, and low $\mathrm{HDL}$ as $\leq 1.03 \mathrm{mmol} / \mathrm{L}$ [17]. Dyslipidemia was diagnosed according to the Guide for Dyslipidemia in China ( $\mathrm{TC}>5.72 \mathrm{mmol} / \mathrm{L}$ and $/$ or $\mathrm{TG}>1.70 \mathrm{mmol} / \mathrm{L}$ and $/$ or $\mathrm{HDL}<0.91 \mathrm{mmol} / \mathrm{L}$ ) [18].

Blood pressure was measured by trained examiners using a mercury sphygmomanometer according to a standard protocol. After each subject rested for at least $5 \mathrm{~min}$ in the sitting position, his or her blood pressure was measured three times on the right arm with the arm cuff maintained at the level of the heart. These three readings were averaged for the analysis. Hypertension was diagnosed if the average of the three blood pressure readings was at least $140 \mathrm{mmHg}$ systolic blood pressure (SBP) or $90 \mathrm{mmHg}$ diastolic blood pressure (DBP), or if the subjects were on antihypertensive medication. Diabetes was diagnosed if the fasting serum glucose concentration was at least $7.0 \mathrm{mmol} / \mathrm{L}$ [19] or if the subject was on antidiabetic medication or insulin treatment [20]. Hyperuricemia was defined as uric acid level $\geq 417 \mu \mathrm{mol} / \mathrm{L}$ in men or $\geq 357 \mu \mathrm{mol} / \mathrm{L}$ in women [21].

Low income was defined as income $<500 \mathrm{RMB}$ per month. Frequent walking was defined as walking for 2 miles or more, at least once a week [22]. A semiquantitative measure of light $(<10$ cigarettes/day), medium (10-20 cigarettes/day) and heavy ( $>20$ cigarettes/day) smoking was performed for a dose-response analysis in the smoking participants. None of the women in the study population smoked, so we did not calculate smoking data for women. The use of oral contraceptives was divided into an exposed group, where the participants had taken pills for one year or more, and an unexposed group, where the participants had taken pills for less than 1 year or not at all.

For database management and statistical analysis, we used SAS software version 9.2 (SAS Institute, Cary, NC, USA). Odds ratios of individual associated factors and their association with OA by sex were calculated by logistic regression analyses. Stepwise logistic regression analyses were used to evaluate the factors that were independently associated with OA in the whole population, and separately in women and men; $P$-values for covariates to be included in the model were set at 0.05 . $P$-values $<0.05$ were considered statistically significant, and all tests were two-tailed [11].

\section{Results}

In total, 3428 individuals aged 40 years and older were identified in the randomly selected households in Gaoyou County, a rural area $300 \mathrm{~km}$ north of Shanghai, China (Fig. 1). Those who declined to participate were older compared with the study participants (mean \pm SD age $58 \pm 9$ years versus $55 \pm 10$ years). Table 1 show the sex and age distribution of the respondents. There were 568 knee OA cases in the study; 279 in females and 289 in

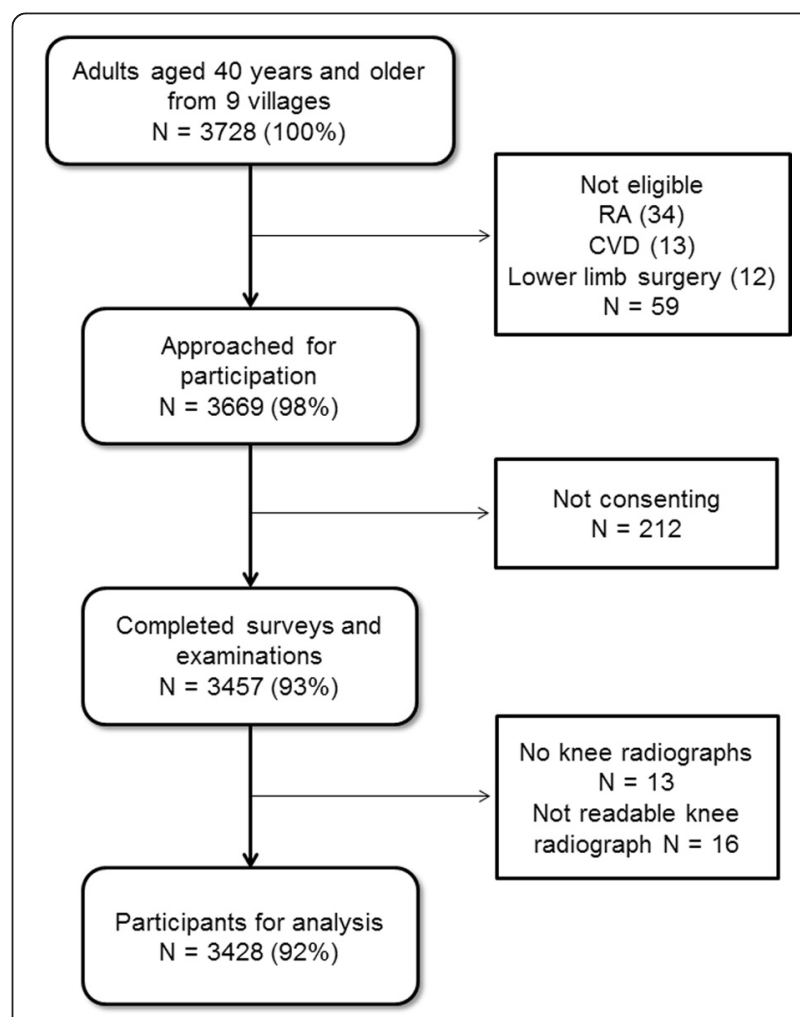

Fig. 1 Gaoyou symptomatic knee osteoarthritis study flow chart. RA = Rheumatoid arthritis. CVD = Cerebrovascular disease 
Table 1 Prevalence of symptomatic knee osteoarthritis stratified by gender and age group in the study population

\begin{tabular}{|c|c|c|c|c|}
\hline \multirow[t]{2}{*}{ Age group } & \multicolumn{2}{|c|}{ Women } & \multicolumn{2}{|l|}{ Men } \\
\hline & $n$ & Case (\%) & $n$ & Case (\%) \\
\hline $40 \sim$ & 730 & 70 (9.59) & 440 & $40(9.09)$ \\
\hline $50 \sim$ & 516 & 89 (17.25) & 497 & 96 (19.32) \\
\hline $60 \sim$ & 415 & $89(21.45)$ & 554 & $111(20.04)$ \\
\hline$\geq 70$ & 106 & 31 (29.25) & 170 & $42(24.71)$ \\
\hline Total & 1767 & 279 (15.79) & 1661 & $289(17.40)$ \\
\hline
\end{tabular}

males. The overall prevalence of OA was $16.57 \%$ ( $15.79 \%$ in women, $17.40 \%$ in men) with no significant sex differences (men: women, odds ratio [OR] $1.12,95 \%$ confidence interval [CI] 0.93-1.34, $P=0.23$ ). The overall prevalence of knee OA increased significantly with age $(P<0.01)$, reaching $29.25 \%$ of women and $24.71 \%$ of men in the age-group 70 years and older. After gender stratification, both male and female knee OA prevalence increased dramatically with age $(P<0.01)$ (Table 1).

Table 2 shows the results of univariate analysis of individual associated factors and their association with OA by sex. Among women, the associated factors significantly associated with OA were age, overweight, central adiposity, high LDLC, high TC, high TG, dyslipidemia, hypertension and low income $(P<0.05)$. Among men, the factors showing a significant association with $\mathrm{OA}$ were age, high LDLC, hypertension, low income and frequent walking $(P<0.05)$. Interestingly, male heavy smokers were less likely to develop severe knee OA compared with nonsmokers $(P<0.05)$. Table 3 shows the results of stepwise logistic regression analysis of associated factors for OA in all the respondents, irrespective of sex. The factors significantly associated with $\mathrm{OA}$ in the general population were age and overweight $(P<0.05)$. Table 4 shows the results of stepwise logistic regression analysis of associated factors for OA by sex. The factors significantly associated with OA were again shown to be age in both women and men $(P<0.05)$. However, overweight was only a factor in women, while high LDLC, hypertension, and frequent walking were only significant factors in men $(P<0.05)$.

\section{Discussion}

Along with the development of the Chinese economy and the associated changes in lifestyle, there is an increase in the aging population and the resulting burden on family and society caused by knee OA has attracted increasing attention. Extensive epidemiological surveys have indicated that the prevalence of knee OA varies greatly with region, race and socioeconomic conditions. Prevalence is also influenced by a variety of environmental and genetic factors. To date, the large-sample epidemiological surveys that have been carried out have mainly concentrated on
North America and European regions. There have been few relevant surveys in China, especially in the middle and lower reaches of the Yangtze River region which has a high population density. We carried out an epidemiological survey of knee OA in China to address this gap in knowledge.

The result indicates that age and weight are positively correlated with the prevalence of knee OA, which is in agreement with the results of most other epidemiological surveys [23, 24]. Lohmander et al. [15] carried out a largesample follow-up survey on discharged patients in Sweden over 10 years. They found that BMI, waist circumference, waist-hip ratio, weight and percentage body fat, which are all closely related to weight, showed a positive correlation with the prevalence of knee OA. Jiang et al. arrived at the conclusion that obesity is a risk factor for knee OA by systematically analyzing the correlation between BMI and knee OA in 21 independent reports [25]. Our result also indicated that the population with high BMI showed a significantly increased prevalence of knee OA, with the increase in waist circumference being closely related to the prevalence of knee OA in females. This may be because the pressure exerted on the articular cartilage increases, which accelerates degeneration. However, some studies have indicated that obesity is also positively correlated with the prevalence of OA in non-load-bearing joints such as in the hand [26]. This means that the load on the joint cannot completely explain the relationship between obesity and knee OA.

Soran et al. [27] found that levels of serum high-density lipoprotein-cholesterol (HDL-C), total thiol (total free sulfhydryl groups, - $\mathrm{SH}$ ), paraoxonase and arylesterase activities were significantly lower in the OA patient group than in controls, while lipid hydroperoxide (LOOH) and low-density lipoprotein (LDL) levels were significantly higher. Dyslipidemia may result in the ectopic deposition of lipids in particular in chondrocytes, which aggravates lipid metabolism disorders in degenerative articular cells and promotes the development of OA. Thus obesityrelated metabolic factors, especially adipokines, can induce the expression and release of inflammatory factors and metabolic enzymes, inhibit the synthesis of articular cartilage and stimulate the remodeling of subchondral bone [5]. Singh et al. found that patients with OA are more likely to have hypertension (40\% vs. $25 \%$ ), diabetes (11\% vs. $6 \%$ ), high total cholesterol (32\% vs. $24 \%$ ), and renal impairment (37 \% vs. $27 \%$ ) compared with the unaffected population [28]. The mechanism may involve ischemia below the cartilage of knee OA patients caused by hypertension. This type of ischemia can inhibit the metabolism of articular cartilage and trigger bone remodeling.

In the late 1980s, the negative correlation between smoking and OA was shown by a cross-sectional epidemiological 
Table 2 Odds ratios (OR) of individual associated factors with symptomatic knee osteoarthritis by sex

\begin{tabular}{|c|c|c|c|c|c|c|}
\hline \multirow[t]{2}{*}{ Associated factor } & \multicolumn{3}{|c|}{ Women } & \multicolumn{3}{|l|}{ Men } \\
\hline & $n$ & Case (\%) & OR & $n$ & Case (\%) & OR \\
\hline \multicolumn{7}{|l|}{ Age } \\
\hline $40 \sim$ & 730 & 70 (9.59) & 1 & 440 & 40 (9.09) & 1 \\
\hline $50 \sim$ & 516 & $89(17.25)$ & $1.97(1.40-2.75)$ & 497 & $96(19.32)$ & $2.39(1.61-3.55)$ \\
\hline $60 \sim$ & 415 & $89(21.45)$ & $2.57(1.83-3.62)$ & 554 & $111(20.04)$ & $2.51(1.70-3.69)$ \\
\hline$\geq 70$ & 106 & $31(29.25)$ & $3.90(2.40-6.33)$ & 170 & $42(24.71)$ & $3.28(2.04-5.28)$ \\
\hline \multicolumn{7}{|l|}{ Overweight } \\
\hline No & 967 & $134(13.86)$ & 1 & 950 & 163 (17.16) & 1 \\
\hline Yes & 800 & $145(18.13)$ & $1.35(1.05-1.74)$ & 711 & $126(17.72)$ & $1.04(0.80-1.34)$ \\
\hline \multicolumn{7}{|l|}{ Central adiposity } \\
\hline No & 1193 & $116(9.72)$ & 1 & 1207 & 202 (16.74) & 1 \\
\hline Yes & 574 & 115 (20.03) & $1.53(1.18-1.99)$ & 454 & 87 (19.16) & $1.18(0.89-1.56)$ \\
\hline \multicolumn{7}{|l|}{ High LDLC } \\
\hline NO & 1656 & $252(15.22)$ & 1 & 1558 & 266 (17.07) & 1 \\
\hline Yes & 111 & $27(24.32)$ & $1.79(1.14-2.82)$ & 103 & $23(22.33)$ & $1.40(1.06-2.26)$ \\
\hline \multicolumn{7}{|l|}{ High TC } \\
\hline NO & 1568 & 239 (15.24) & 1 & 1493 & 256 (17.15) & 1 \\
\hline Yes & 199 & $42(21.11)$ & $1.44(1.00-2.08)$ & 168 & 33 (19.64) & $1.18(0.79-1.77)$ \\
\hline \multicolumn{7}{|l|}{ High TG } \\
\hline NO & 1518 & 228 (15.02) & 1 & 1391 & 247 (17.76) & 1 \\
\hline Yes & 249 & $53(21.29)$ & $1.49(1.07-2.08)$ & 270 & $42(15.56)$ & $0.85(0.60-1.22)$ \\
\hline \multicolumn{7}{|l|}{ Low HDL } \\
\hline NO & 1457 & $236(16.20)$ & 1 & 1250 & 237 (18.96) & 1 \\
\hline Yes & 310 & $54(17.41)$ & $0.84(0.66-1.32)$ & 411 & $68(16.55)$ & $0.65(0.44-1.25)$ \\
\hline \multicolumn{7}{|l|}{ Dyslipidemia } \\
\hline NO & 1181 & 169 (14.31) & 1 & 1024 & 191 (18.65) & 1 \\
\hline Yes & 586 & $111(18.94)$ & $1.40(1.08-1.82)$ & 637 & 98 (15.38) & $0.79(0.61-1.04)$ \\
\hline \multicolumn{7}{|l|}{ Hypertension } \\
\hline No & 903 & $122(13.51)$ & & 652 & $92(14.11)$ & \\
\hline Yes & 864 & 157 (18.17) & $1.42(1.10-1.84)$ & 1009 & 197 (19.52) & $1.48(1.13-1.93)$ \\
\hline \multicolumn{7}{|l|}{ Diabetes } \\
\hline No & 1639 & 257 (15.68) & & 1539 & 270 (17.54) & \\
\hline Yes & 128 & $22(17.19)$ & $1.12(0.69-1.80)$ & 122 & $19(15.57)$ & $0.87(0.52-1.44)$ \\
\hline \multicolumn{7}{|l|}{ Hyperuricemia } \\
\hline No & 1603 & $251(15.66)$ & & 1412 & 247 (17.49) & \\
\hline Yes & 164 & $28(17.07)$ & $1.11(0.72-1.70)$ & 249 & $42(16.87)$ & $0.96(0.67-1.37)$ \\
\hline \multicolumn{7}{|l|}{ Low income } \\
\hline NO & 1571 & $238(15.15)$ & & 1453 & 239 (16.45) & \\
\hline Yes & 196 & $43(21.94)$ & $1.54(1.06-2.19)$ & 208 & $50(24.04)$ & $1.61(1.14-2.27)$ \\
\hline \multicolumn{7}{|l|}{ Often walk } \\
\hline No & 1545 & $242(15.66)$ & & 1444 & 241 (16.69) & 1 \\
\hline Yes & 222 & $37(16.67)$ & $1.08(0.74-1.57)$ & 217 & $48(22.12)$ & $1.42(1.00-2.01)$ \\
\hline
\end{tabular}


Table 2 Odds ratios (OR) of individual associated factors with symptomatic knee osteoarthritis by sex (Continued)

\begin{tabular}{|c|c|c|c|c|c|c|}
\hline \multicolumn{7}{|l|}{ Smoke } \\
\hline Nonsmoker & & & & 487 & $92(18.89)$ & 1 \\
\hline$<10$ per/day & & & & 241 & $46(19.09)$ & $0.91(0.71-1.34)$ \\
\hline $10 \sim$ per/day & & & & 405 & $72(17.78)$ & $0.82(0.66-1.21)$ \\
\hline $20 \sim$ per/day & & & & 528 & $67(12.69)$ & $0.62(0.45-0.86)$ \\
\hline \multicolumn{7}{|c|}{ Oral Contraceptive Users } \\
\hline No & 1644 & $263(16.00)$ & 1 & 0 & & \\
\hline Yes & 123 & $16(13.01)$ & $0.79(0.46-1.35)$ & 0 & & \\
\hline
\end{tabular}

LDLC low-density lipoprotein cholesterol, TC total cholesterol, TG triglycerides, $H D L$ high density lipoprotein

study and a cohort study by Felson. Later, Felson et al. continued to investigate these respondents. X-ray images of the knee joint in the load-bearing position were taken in 1983-1985 and again in 1992-1993. The results indicated that the prevalence of $\mathrm{OA}$ in the smokers was lower compared with the non-smokers $(\mathrm{OR}=0.4,95 \% \mathrm{CI}[0.2-0.8])$ $[13,14]$. In the past 20 years, several research institutes have been observing the relationship between smoking and OA. Samanta et al. [29] found a negative correlation between smoking and large-joint $\mathrm{OA}$ according to the data from the Nottingham Clinical Center Database (UK). Jarvholm et al. [24] found a negative correlation between smoking and hip OA in a study of male construction workers in Sweden, but the influence of smoking was weaker than that of BMI and age.

However, some reports suggest that smoking is not obviously related to OA. In 2011, Hui et al. [30] performed a meta-analysis of the existing data concerning smoking and OA. They reported that the results indicative of a negative correlation between smoking and OA were mainly derived from a hospital setting. The protective effect of smoking on the joints needs to be further supported by a normatively designed survey with community-based data. In our survey, data were collected from the community for multi-factor regression analysis, and the results indicate smoking might be associated with a lower prevalence of OA in male smokers according to univariate analysis.

There are several theories concerning the mechanism underlying the protective effect of smoking: 1) nicotine can increase the glycosaminoglycan level and collagen synthesis activity in articular cartilage; 2) nicotine can regulate the anabolic activity of articular cartilage; 3 ) nicotine can influence the deformability of subchondral bone

Table 3 Stepwise logistic regression analysis of factors associated with symptomatic knee osteoarthritis in the entire study population (both sexes combined)

\begin{tabular}{llr}
\hline Variable & OR $(95 \% \mathrm{Cl})$ & $P$ value \\
\hline Age & $1.04(1.03-1.05)$ & $<0.0001$ \\
Overweight & $1.05(1.02-1.08)$ & 0.0024 \\
\hline
\end{tabular}

under impact load by reducing bone density, and therefore reducing the pressure on the cartilage; 4) nicotine can inhibit the inflammatory factors involved in knee OA $[31,32]$. It is notable that surveys from several institutes as well as some meta-analyses have indicated that smoking is a risk factor for rheumatoid arthritis, especially for patients with positive rheumatoid factor $[33,34]$. Evidently the relevant mechanism between smoking and knee arthritis, such as OA and rheumatoid arthritis, needs to be further explored.

Our study also found that low income and long-term walking increase the prevalence of knee OA. This may be because the low-income population usually engages in heavy physical labor, which increases the load on the knee joints. Similarly, long-term walking also increases loading on the knee joints, thus aggravating any damage to the cartilage [35]. No correlation between oral contraceptive use and knee OA was found in our survey. There have been several studies indicating a possible inverse relationship between estrogen intake and knee OA, but in a study by Sandmark et al., estrogen therapy for women over 50 was associated with an increased relative risk of 1.8 (95 \% CI 1.2-2.6), while use of oral contraceptives did not influence the risk [23].

Our study has some limitations. First, radiological examination and symptomatic examination are the common diagnostic methods for the epidemiological investigation of knee OA. In our study, the symptomatic diagnosis method was used as the main inclusion criterion for knee

Table 4 Stepwise logistic regression analysis of factors associated with symptomatic knee osteoarthritis by sex

\begin{tabular}{llllll}
\hline Variable & Women & & & Men & \\
\cline { 2 - 3 } & OR $(95 \% \mathrm{Cl})$ & $P$ value & & OR $(95 \% \mathrm{Cl})$ & $P$ values \\
\hline Age & $1.05(1.03-1.06)$ & $<0.0001$ & & $1.03(1.02-1.05)$ & $<0.0001$ \\
Overweight & $1.05(1.01-1.09)$ & 0.0228 & & \\
High LDLC & & & $1.22(1.01-1.46)$ & 0.0362 \\
Hypertension & & & $1.01(1.00-1.01)$ & 0.0211 \\
Often Walk & & & $1.57(1.10-2.24)$ & 0.0128 \\
\hline
\end{tabular}

LDLC low-density lipoprotein cholesterol 
OA, so a questionnaire was the primary screening tool. Inevitably, there were likely to be some differences in the discrimination of information by investigation staff although they had all received the same training. Second, the survey data were epidemiological data from only one small area. More epidemiological data from different areas need to be collected and analyzed in future. Third, the causality between $\mathrm{OA}$ and associated factors cannot be confirmed fully by the cross-sectional study method, the results need to be further confirmed by prospective studies.

\section{Conclusions}

In conclusion, aging, obesity, increased knee joint movement, low income and relevant multiple metabolic disorders are important associated factors for OA. Smoking seems to be associated with a lower prevalence of OA, although it has not been included in stepwise logistic regression analysis. Considering the difficulty of epidemiological investigation in rural populations and the availability of study data in this region, we believe the results of our population-based screening study can aid in international comparisons and elucidate the factors associated with knee OA in China.

\section{Abbreviations}

BMI: body mass index; DBP: diastolic blood pressure; HDL: high-density lipoprotein; K/L: Kellgren/Lawrence; LDLC: low-density lipoprotein cholesterol; MS: metabolic syndrome; OA: osteoarthritis; SBP: systolic blood pressure; TC: total cholesterol; TG: triglycerides.

\section{Competing interests}

The authors declare that they have no competing interests.

\section{Authors' contributions}

$Y L, H F Z$, and $J H$ carried out the studies and data analyses and drafted the manuscript. ZJY and ZJW carried out the data collection. NXL, JL, ZHH, and WMF conceived the study, and participated in its design and coordination. All authors read and approved the final manuscript.

\section{Acknowledgements}

This study was supported by grants from the National Natural Science Foundation of China (Nos. 81373397 and 81341017), the Jiangsu Department of Science and Technology Basic Research Program (No. BK20131443), the State Scholarship Fund (No. 201208320286), and the National Key Technologies R\&D Program of China during the 11th Five-Year Plan Period (No. 2007BAI07A10)

\section{Author details}

${ }^{1}$ Department of Orthopedics, The First Affiliated Hospital of Nanjing Medical University, Guang Zhou Road 300, Nanjing 210029, China. ²Department of Infectious Diseases, The First Affiliated Hospital of Nanjing Medical University, Nanjing 210029, China. ${ }^{3}$ Department of Cardiology, The First Affiliated Hospital of Nanjing Medical University, Nanjing 210029, China. ${ }^{4}$ Centre Clinics of Baqiao, Gaoyou 225642, China.

Received: 29 May 2015 Accepted: 26 January 2016

Published online: 30 January 2016

\section{References}

1. Shen J, Chen D. Recent progress in osteoarthritis research. J Am Acad Orthop Surg. 2014;22(7):467-8.

2. Zhang Y, Xu L, Nevitt MC, Aliabadi P, Yu W, Qin M, et al. Comparison of the prevalence of knee osteoarthritis between the elderly Chinese population in
Beijing and whites in the United States: The Beijing Osteoarthritis Study. Arthritis Rheum. 2001;44(9):2065-71.

3. Kang $X$, Fransen M, Zhang Y, Li H, Ke Y, Lu M, et al. The high prevalence of knee osteoarthritis in a rural Chinese population: the Wuchuan osteoarthritis study. Arthritis Rheum. 2009;61(5):641-7.

4. Lin J, Fransen M, Kang X, Li H, Ke Y, Wang Z, et al. Marked disability and high use of nonsteroidal antiinflammatory drugs associated with knee osteoarthritis in rural China: a cross-sectional population-based survey. Arthritis Res Ther. 2010;12(6):R225.

5. Zhuo Q, Yang W, Chen J, Wang Y. Metabolic syndrome meets osteoarthritis. Nat Rev Rheumatol. 2012;8(12):729-37.

6. Lee S, Kim TN, Kim SH, Kim YG, Lee CK, Moon HB, et al. Obesity, metabolic abnormality, and knee osteoarthritis: A cross-sectional study in Korean women. Mod Rheumatol. 2015;25(2):292-7.

7. Katz JD, Agrawal S, Velasquez M. Getting to the heart of the matter: osteoarthritis takes its place as part of the metabolic syndrome. Curr Opin Rheumatol. 2010:22(5):512-9.

8. Berenbaum F, Eymard F, Houard X. Osteoarthritis, inflammation and obesity. Curr Opin Rheumatol. 2013;25(1):114-8.

9. Gandhi R, Razak F, Tso P, Davey JR, Mahomed NN. Asian ethnicity and the prevalence of metabolic syndrome in the osteoarthritic total knee arthroplasty population. J Arthroplasty. 2010;25(3):416-9.

10. Yao WM, Zhang HF, Zhu ZY, Zhou YL, Liang NX, Xu DJ, et al. Genetically elevated levels of circulating triglycerides and brachial-ankle pulse wave velocity in a Chinese population. J Hum Hypertens. 2013; 27(4):265-70

11. Zhu Z, Zhang H, Yao W, Liang N, Gong L, Yin Z, et al. Physical activity modifies the association between CYBA gene polymorphisms and small artery elasticity in a Chinese population. Hypertens Res. 2012:35(7):739-44.

12. Duddy J, Kirwan JR, Szebenyi B, Clarke S, Granell R, Volkov S. A comparison of the semiflexed (MTP) view with the standing extended view (SEV) in the radiographic assessment of knee osteoarthritis in a busy routine $\mathrm{X}$-ray department. Rheumatology (Oxford). 2005;44(3):349-51.

13. Felson DT, Anderson JJ, Naimark A, Kannel W, Meenan RF. The prevalence of chondrocalcinosis in the elderly and its association with knee osteoarthritis: the Framingham Study. J Rheumatol. 1989;16(9):1241-5.

14. Felson DT, Naimark A, Anderson J, Kazis L, Castelli W, Meenan RF. The prevalence of knee osteoarthritis in the elderly. The Framingham Osteoarthritis Study. Arthritis Rheum. 1987;30(8):914-8.

15. Lohmander LS, de Verdier MG, Rollof J, Nilsson PM, Engstrom G. Incidence of severe knee and hip osteoarthritis in relation to different measures of body mass: a population-based prospective cohort study. Ann Rheum Dis. 2009:68(4):490-6.

16. Ford ES. Prevalence of the metabolic syndrome defined by the International Diabetes Federation among adults in the U.S. Diabetes Care. 2005;28(11):2745-9.

17. Zhang ZQ, Deng J, He LP, Ling WH, Su YX, Chen YM. Comparison of various anthropometric and body fat indices in identifying cardiometabolic disturbances in Chinese men and women. PLoS One. 2013:8(8):e70893.

18. Chen $L$, Lei $L$, Jin $T$, Nordberg M, Nordberg GF. Plasma metallothionein antibody, urinary cadmium, and renal dysfunction in a Chinese type 2 diabetic population. Diabetes Care. 2006;29(12):2682-7.

19. Schwartz SS, Epstein S, Corkey BE, Grant SF, Gavin JR 3rd, Aguilar RB. The Time Is Right for a New Classification System for Diabetes: Rationale and Implications of the beta-Cell-Centric Classification Schema. Diabetes Care. 2016:39(2):179-86.

20. Boehme MW, Buechele G, Frankenhauser-Mannuss J, Mueller J, Lump D, Boehm BO, et al. Prevalence, incidence and concomitant co-morbidities of type 2 diabetes mellitus in South Western Germany-a retrospective cohort and case control study in claims data of a large statutory health insurance. BMC Public Health. 2015;15:855.

21. Darmawan J, Valkenburg HA, Muirden KD, Wigley RD. The epidemiology of gout and hyperuricemia in a rural population of Java. J Rheumatol. 1992;19(10):1595-9.

22. Sutton AJ, Muir KR, Mockett S, Fentem P. A case-control study to investigate the relation between low and moderate levels of physical activity and osteoarthritis of the knee using data collected as part of the Allied Dunbar National Fitness Survey. Ann Rheum Dis. 2001;60(8):756-64.

23. Sandmark H, Hogstedt C, Lewold S, Vingard E. Osteoarthrosis of the knee in men and women in association with overweight, smoking, and hormone therapy. Ann Rheum Dis. 1999;58(3):151-5. 
24. Jarvholm B, Lewold S, Malchau H, Vingard E. Age, bodyweight, smoking habits and the risk of severe osteoarthritis in the hip and knee in men. Eur J Epidemiol. 2005;20(6):537-42.

25. Jiang L, Tian W, Wang Y, Rong J, Bao C, Liu Y, et al. Body mass index and susceptibility to knee osteoarthritis: a systematic review and meta-analysis. Joint Bone Spine. 2012;79(3):291-7.

26. Koonce RC, Bravman JT. Obesity and osteoarthritis: more than just wear and tear. J Am Acad Orthop Surg. 2013;21(3):161-9.

27. Soran N, Altindag O, Cakir H, Celik H, Demirkol A, Aksoy N. Assessment of paraoxonase activities in patients with knee osteoarthritis. Redox Rep. 2008:13(5):194-8.

28. Singh G, Miller JD, Lee FH, Pettitt D, Russell MW. Prevalence of cardiovascular disease risk factors among US adults with self-reported osteoarthritis: data from the Third National Health and Nutrition Examination Survey. Am J Manag Care. 2002:8(15 Suppl):S383-91.

29. Samanta A, Jones A, Regan M, Wilson S, Doherty M. Is osteoarthritis in women affected by hormonal changes or smoking. Br J Rheumatol. 1993;32(5):366-70

30. Hui M, Doherty M, Zhang W. Does smoking protect against osteoarthritis? Meta-analysis of observational studies. Ann Rheum Dis. 2011;70(7):1231-7.

31. Elloumi M, Kallel MH. Which relationship does osteoarthritis share with smoking. Osteoarthritis Cartilage. 2007;15(9):1097-8.

32. van Maanen MA, Lebre MC, van der Poll T, LaRosa GJ, Elbaum D, Vervoordeldonk MJ, et al. Stimulation of nicotinic acetylcholine receptors attenuates collagen-induced arthritis in mice. Arthritis Rheum. 2009:60(1):114-22.

33. Di GD, Discacciati A, Orsini N, Wolk A. Cigarette smoking and risk of rheumatoid arthritis: a dose-response meta-analysis. Arthritis Res Ther 2014;16(2):R61.

34. Sugiyama D, Nishimura K, Tamaki K, Tsuji G, Nakazawa T, Morinobu A, et al. Impact of smoking as a risk factor for developing rheumatoid arthritis: a meta-analysis of observational studies. Ann Rheum Dis. 2010;69(1):70-81.

35. Veenhof $C$, Huisman PA, Barten JA, Takken T, Pisters MF. Factors associated with physical activity in patients with osteoarthritis of the hip or knee: a systematic review. Osteoarthritis Cartilage. 2012;20(1):6-12.

\section{Submit your next manuscript to BioMed Central and we will help you at every step:}

- We accept pre-submission inquiries

- Our selector tool helps you to find the most relevant journal

- We provide round the clock customer support

- Convenient online submission

- Thorough peer review

- Inclusion in PubMed and all major indexing services

- Maximum visibility for your research

Submit your manuscript at www.biomedcentral.com/submit

) Biomed Central 